\title{
Menus de substitution dans les cantines scolaires : de la laïcité à l'intérêt supérieur de l'enfant
}

\section{Anne Fornerod}

\section{(2) OpenEdition}

\section{Journals}

Édition électronique

URL : http://journals.openedition.org/rdr/401

DOI : $10.4000 /$ rdr.401

ISSN : 2534-7462

\section{Éditeur}

Presses universitaires de Strasbourg

Édition imprimée

Date de publication : 24 avril 2018

Pagination : 181-188

ISBN : 979-10-344-0017-1

ISSN : 2493-8637

\section{Référence électronique}

Anne Fornerod, «Menus de substitution dans les cantines scolaires : de la laïcité à l'intérêt supérieur de l'enfant », Revue du droit des religions [En ligne], 5 | 2018, mis en ligne le 25 novembre 2019, consulté le 19 novembre 2020. URL : http://journals.openedition.org/rdr/401 ; DOI : https://doi.org/10.4000/rdr. 401

La revue du droit des religions est mise à disposition selon les termes de la Creative Commons Attribution - Pas d'Utilisation Commerciale 4.0 International - CC BY-NC 4.0. 


\section{MENUS DESUBSTITUTION DANSLES CANTINES SCOLAIRES:DELALÄ̈CITÉÁL'INTÉRÊT SUPÉRIEURDEL'ENFANT}

\section{Anne FORNEROD}

Université de Strasbourg/CNRS, Droit, Religion, Entreprise et Société (DRES)

$\mathrm{D}$ ans un jugement du 28 août 2017, le tribunal administratif de Dijon s'est à nouveau prononcé sur la décision prise par le maire de Chalonsur-Saône de ne plus proposer de menus de substitution dès lors qu'un plat contenant du porc serait servi dans les cantines scolaires de la ville ${ }^{1}$. Précédemment, dans une ordonnance du 12 août $2015^{2}$, le tribunal administratif de Dijon, saisi cette fois dans le cadre d'un référé suspension, avait rejeté la requête de la Ligue de défense judiciaire des musulmans après avoir estimé que l'accès à la cantine des enfants de confession musulmane n'était pas compromis. Deux ans plus tard, infirmant l'ordonnance rendue précédemment, le tribunal a considéré l'affaire à l'aune de la Convention internationale des droits de l'enfant et conclu que «dans les circonstances particulières de l'espèce, les décisions attaquées, même si l'information des familles a été prévue avant puis pendant la mise en œuvre de la délibération attaquée, ne peuvent pas être regardées comme ayant accordé une attention primordiale à l'intérêt supérieur des enfants». Les décisions de la commune relatives aux menus scolaires sont donc annulées.

1. TA Dijon, 28 août 2017, n 1502100, 1502726, Ligue de défense judiciaire des musulmans et $a$. 2. TA Dijon, 12 août 2015, n 1502101, Ligue de défense judiciaire des musulmans. 
La question des menus de substitution dans les cantines relève de la thématique générale des pratiques religieuses alimentaires et en l'occurrence celles qui s'appuient sur l'interdiction de consommer de la viande de porc - sont ici concernées les religions juive et musulmane. Ces pratiques présentent une double singularité lorsqu'elles revêtent une dimension collective et qu'elles s'expriment dans le cadre d'un service public. S'agissant ici des cantines scolaires, le cadre légal s'avère relativement restreint (1), rendant d'autant plus intéressante la décision du tribunal administratif de Dijon de s'appuyer sur la Convention internationale des droits de l'enfant pour trancher le litige (2).

\section{PRATIQUES RELIGIEUSES ALIMENTAIRES ET CANTINES SCOLAIRES}

La question des menus de substitution dans les cantines scolaires ne relève pas de la «question scolaire» proprement dite, même si les cantines participent d'une certaine façon de l'éducation alimentaire et de la sensibilisation à la gastronomie ${ }^{3}$. Les cantines scolaires constituent en effet un service public facultatif, non strictement lié au service public de l'enseignement ${ }^{4}$. Il est de ce point de vue significatif que la question soit traitée dans une circulaire du ministère de l'Intérieur de 2011 relative aux demandes de régimes alimentaires particuliers dans les services de restauration collective du service public ${ }^{5}$. Comme l'indique clairement ce texte, «la loi du 13 août 2004 relative aux libertés et responsabilités locales transfère aux collectivités locales la responsabilité de la restauration scolaire. [...] En l'absence de réglementation nationale précise, il appartient à chaque organe délibérant compétent (conseil municipal pour l'enseignement primaire, conseil général pour les collèges et conseil régional pour les lycées) de poser des règles en la matière».

Les menus de substitution s'insèrent donc dans un ensemble de considérations touchant à l'accès même aux cantines (la politique tarifaire notamment) et à l'alimentation proposée (équilibre nutritionnel, santé des élèves...). Les

3. V. en ce sens la question parlementaire $n^{\circ} 32420$ : JOAN, 16 juill. 2013, p. 7372 ou encore la question écrite $\mathrm{n}^{\circ} 18565$ : JO Sénat, 29 oct. 2015, p. 2521.

4. V. le rapport du Défenseur des droits, L'égal accès des enfants à la cantine de l'école primaire, 28 mars 2013, disponible en ligne: http://www.ladocumentationfrancaise.fr/var/ storage/rapports-publics/134000207.pdf [consulté le 17 janv. 2018].

5. Circulaire NOR/IOCK1110778C du 16 août 2011. Rappel des règles afférentes au principe de laïcité - Demandes de régimes alimentaires particuliers dans les services de restauration collective du service public: http://www.legirel.cnrs.fr/spip.php?article84 [consulté le 17 janv. 2018]. 
collectivités ne sont pas exemptes d'obligations dans la mesure où elles doivent garantir l'égal accès à la cantine au titre du principe général d'égal accès aux services publics et, s'agissant de l'alimentation elle-même, respecter «l'objectif d'équilibre nutritionnel des repas servis par les services de restauration scolaire» posé par le décret $n^{\circ}$ 2011-1227 du 30 septembre 2011. De là, comment s'élaborent les réponses aux demandes d'adaptation des menus motivées par des convictions religieuses? L'on observe que les menus de substitution sont appréhendés nettement - voire uniquement - en fonction de leur lien avec des pratiques religieuses et indépendamment d'autres problématiques qui touchent les cantines scolaires. À titre d'exemple, et concernant l'aspect nutritionnel, le rapprochement proposé entre la question des repas végétariens et les menus de substitution, en ce que les premiers offriraient une solution aux attentes soulevées par les seconds, n'a pas été suivi d'effets ${ }^{6}$.

De là, le cadre juridique des menus de substitution dans les cantines scolaires s'avère très succinct. Le jugement du tribunal administratif résume assez clairement l'état du droit en affirmant que «l'obligation de proposer aux enfants un menu de substitution ne résulte d'aucune disposition constitutionnelle, législative ou réglementaire et d'aucun principe». L'absence d'obligation des collectivités locales de répondre aux demandes d'adaptation

6. Dans une réponse à une question parlementaire $\left(n^{\circ} 18565\right)$ faisant observer que «la mise en place d'un repas végétarien de substitution au menu quotidien aurait pour conséquence directe de faire chuter les volumes de viande consommée dans les restaurants scolaires», le ministère de l'Agriculture, de l'Agroalimentaire et de la Forêt décèle une référence à une "proposition de loi de M. Yves Jégo, récemment déposée à l'Assemblée nationale, relative à l'instauration en restauration scolaire d'un menu végétarien en alternative au menu quotidien». Selon le ministère, «si son objectif est clairement de traiter la question des menus confessionnels, elle le fait en modifiant les dispositions législatives relatives aux règles nutritionnelles en restauration scolaire. Au-delà du caractère inapproprié de la base législative choisie, cette initiative instrumentalise la question de l'équilibre nutritionnel des menus à la cantine, sujet de santé publique d'importance, pour traiter d'un thème de société particulièrement sensible»: JO Sénat, 10 déc. 2015, p. 3372.

Plus récemment, un député faisait remarquer, à propos de la réduction des protéines carnées dans les cantines scolaires, qu'« incidemment, cette alternative [végétarienne] permettrait de régler définitivement l'instrumentalisation politicienne de la question des menus scolaires. Les plats végétariens permettraient en respectant la laïcité, de permettre à tous les enfants d'avoir une alimentation suffisamment protéinée lors des repas scolaires, indépendamment de leurs éventuels interdits alimentaires liés à une religion». Sans répondre précisément sur ce point mais en faisant preuve d'un certain assouplissement, le ministère de l'Agriculture et de l'Alimentation indique tout de même que «seuls huit repas sur vingt doivent donc comporter du poisson, de la viande ou des abats. Aucune disposition particulière quant à la nature du plat protidique ne s'applique pour les autres repas, les protéines pouvant être issues de viandes, poissons, œufs, abats ou fromages. Une alternative végétarienne peut ainsi être proposée dans le respect des dispositions réglementaires actuelles »: Rép. min. n 1302: JOAN, 31 oct. 2017, p. 5290. 
des menus aux convictions religieuses des élèves ne signifie pas pour autant qu'elles disposent d'une totale discrétionnarité. En effet, le libre choix dont jouissent ici les collectivités territoriales s'exerce entre les limites touchant au respect du principe de laïcité des services publics d'un côté et au respect des convictions religieuses des usagers de l'autre. Le cadre juridique, constitué pour l'essentiel de textes infra-réglementaires, est celui qui vaut pour le principe de laïcité dans les services publics. Ainsi, la circulaire du ministère de l'Intérieur du 16 août 2011, portant précisément sur les demandes de régimes alimentaires particuliers dans les services de restauration collective du service public, renvoie à la Charte de la laïcité dans les services publics selon laquelle «les usagers du service public ne peuvent exiger une adaptation du fonctionnement d'un service public ou d'un équipement public. Cependant, le service s'efforce de prendre en compte les convictions des usagers dans le respect des règles auquel il est soumis et de son bon fonctionnement ${ }^{7} »$. Un tel équilibre vaut également alors même que des dispositions spécifiques existent. Tel est le cas pour les détenus, le Code de procédure pénale contenant des dispositions ${ }^{8}$ "qui visent à permettre l'exercice par les personnes détenues de leurs convictions religieuses en matière d'alimentation sans toutefois imposer à l'administration de garantir, en toute circonstance, une alimentation respectant ces convictions ${ }^{9} »$.

À ces éléments d'ordre infra-réglementaire s'ajoute une jurisprudence très restreinte. Il n'existe a priori qu'un seul précédent au cas de Chalon-surSaône. Le tribunal administratif de Marseille avait, dans un jugement du 26 novembre 1996, refusé d'annuler la délibération du conseil municipal de la ville de Marignane qui modifiait le règlement des cantines scolaires dans le sens d'un refus de suivre un régime alimentaire particulier. Il ne pouvait en conséquence être enjoint à la commune de fournir des menus adaptés aux pratiques religieuses - en l'occurrence des menus sans viande de porc ${ }^{10}$. Dans une espèce plus récente mais moins probante ici, la requérante soutenait, entre autres, que «la composition des repas servis dans les cantines

7. Circulaire NOR/IOCK1110778C du 16 août 2011, précit., p. 2.

8. Aux termes du premier alinéa de l'article 9 du règlement type des établissements pénitentiaires annexé à l'article R. 57-6-18 du Code de procédure pénale, relatif à l'alimentation des personnes détenues: «Chaque personne détenue reçoit une alimentation variée, bien préparée et présentée, répondant tant en ce qui concerne la qualité que la quantité aux règles de la diététique et de l'hygiène, compte tenu de son âge, de son état de santé, de la nature de son travail et, dans toute la mesure du possible, de ses convictions philosophiques ou religieuses.»

9. CE, 25 févr. 2015, n 375724. V., dans le même sens, CE, 10 févr. 2016, n 385929.

10. TA Marseille, 26 nov. 1996, Mme Zitouni c/ Commune de Marignane: D. 1997, IR, p. 30. 
scolaires de la commune d'Orange portait atteinte à la liberté religieuse en ce que, ne comportant pas de viande le vendredi, elle privilégiait les enfants de confession chrétienne». Sans réelle surprise au regard de la liberté de décision dont jouissent les communes en la matière, le Conseil d'État a jugé que «les dispositions relatives aux menus qui ne font référence à aucun interdit alimentaire ne présentent pas non plus un caractère discriminatoire en fonction de la religion des enfants ou de leurs parents ${ }^{11} »$. Il est vrai en outre que le plat de poisson le vendredi correspond à une «tradition» qui ne répond pas à des demandes explicites d'usagers des cantines.

Pour se situer en dehors des rapports classiques entre école publique et laïcité, les cantines scolaires ne demeurent pas à l'écart des discussions autour de la portée de cette dernière. S'inscrivant plutôt dans le registre général de la laicité, la question des menus de substitution dans les cantines scolaires ressurgit régulièrement dans le débat public, médiatique ou parlementaire, en décalage, d'ailleurs, avec la faible part qu'elle occupe en réalité dans les difficultés qui entourent la gestion des cantines ${ }^{12}$. La circulaire du 16 août 2011 laisse de ce point de vue entendre que les situations problématiques sont rares en la matière en précisant «qu'en pratique, la plupart des cantines proposent depuis longtemps des substituts au porc, et servent du poisson le vendredi, permettant ainsi le respect de certaines prescriptions ou recommandations religieuses». Face à des pratiques anciennes et courantes consistant à proposer des menus de substitution et un état du droit assez sommaire, les préconisations en matière de menus de substitution peuvent difficilement aller au-delà du rappel des principes généraux en balance dès lors qu'il s'agit d'articuler neutralité du service public et liberté de religion des usagers. Or, à l'instar d'autres situations où les usagers de services publics demandent une prise en compte de leurs convictions religieuses ${ }^{13}$, l'équilibre entre ces principes s'atteint par l'observation d'un certain pragmatisme dans la définition des solutions. Cette logique de pragmatisme peut se heurter à une approche de la neutralité religieuse entendue largement et dans un sens prohibitif, approche qui sous-tendait la décision de la commune de Chalon-sur-Saône ${ }^{14}$. C'est alors que le biais de la Convention internationale

11. CE, 25 oct. 2002, n 251161, Commune d'Orange.

12. C'est ce qui ressort du rapport établi par le Défenseur des droits, précit.

13. Dans une même logique et dans le respect de l'obligation de neutralité religieuse qui pèse sur eux, les agents publics peuvent faire valoir leur droit à la liberté de religion dans certaines situations. L'on pense ici aux autorisations d'absence pour les fêtes religieuses.

14. V. A. RAmel, "Laïcité et cantines scolaires: les collectivités seules face au choix des menus», La Gazette, 25 oct. 2010, p. 54. 
des droits de l'enfant (CIDE) pris par le tribunal administratif de Dijon en août 2017 apparaît comme original, en ce qu'il dépasse cette tension entre deux acceptions de la laïcité.

\section{LA CONVENTION INTERNATIONALE DES DROITS DE L'ENFANT AU SOUTIEN DES MENUS DE SUBSTITUTION}

À première vue, la prise en considération du respect de prescriptions alimentaires par les usagers d'un service public, fût-il facultatif, relève d'une problématique classique de liberté de religion. C'est ce qui ressort de la jurisprudence administrative relative aux menus confessionnels dans les établissements pénitentiaires. Ainsi le Conseil d'État a-t-il jugé que «l'observation de prescriptions alimentaires peut être regardée comme une manifestation directe de croyances et pratiques religieuses au sens de l'article 9 de la convention européenne de sauvegarde des droits de l'homme et des libertés fondamentales ${ }^{15} »$. Le principe de laïcité, qui trouve dans l'enceinte des établissements d'enseignement public un lieu d'expression emblématique et spécifique, doit donc ici être considéré sous un angle différent au sens où, hors d'un lien avec l'éducation, il requiert la recherche d'un équilibre, somme toute classique dès lors qu'est en cause l'exercice de pratiques religieuses dans le cadre d'un service public, entre la neutralité des pouvoirs publics dans le domaine religieux et le respect de la liberté de religion des personnes. Or, ce n'est pas directement sur le fondement du principe de laïcité que s'est opéré principalement le raisonnement du juge administratif dans cette espèce et l'article 9 de la Convention européenne des droits de l'homme n'est pas visé. La décision municipale «consistant à mettre fin à une telle pratique [les menus de substitution] affecte de manière suffisamment directe et certaine la situation des enfants fréquentant une cantine scolaire et constitue ainsi une décision dans l'appréciation de laquelle son auteur doit, en vertu de l'article 3-1 de la CIDE, accorder une attention primordiale à l'intérêt supérieur de l'enfant ${ }^{16} »$. Habituellement invoquée dans les litiges familiaux ${ }^{17}$, la

15. CE, 25 févr. $2015, \mathrm{n}^{\circ} 375724$.

16. Article 3 \& 1 CIDE: «Dans toutes les décisions qui concernent les enfants, qu'elles soient le fait des institutions publiques ou privées de protection sociale, des tribunaux, des autorités administratives ou des organes législatifs, l'intérêt supérieur de l'enfant doit être une considération primordiale.»

17. Sur la CIDE de manière générale, V. F. Messner, P.-H. PréLot, J.-M. Woehrling, Traité de droit français des religions, Paris LexisNexis, $2^{\mathrm{e}}$ éd. 2013, p. 463 et, dans le cadre des litiges familiaux, p. 1020 et s. 
CIDE et plus spécifiquement son article $3 \S 1$, renvoie à la notion clé d'intérêt supérieur de l'enfant. Il s'ensuit qu'en l'espèce, si les enfants apparaissent comme des usagers à part, ce n'est pas en raison de la nécessité de protéger la «fragile» liberté de conscience des élèves dans le cadre du service public de l'enseignement. L'invocation de la CIDE conduit à une approche en surplomb, l'articulation entre la laïcité - telle qu'invoquée par le maire de la commune du moins - et la liberté de conscience venant au second plan.

L'on s'abstiendra de déduire de ce jugement une solution de principe applicable à l'ensemble des cantines scolaires. Comme le rappelle le tribunal administratif de Dijon, l'invocation de l'article $3 \& 1$ de la Convention internationale des droits de l'enfant suppose une mise en balance des intérêts en présence. En l'espèce, l'ancienneté de la pratique consistant à proposer de tels menus semble avoir été déterminante et ce d'autant plus que son interruption obéissait à une motivation rejetée par le tribunal. Plus précisément, ont été mis en perspective par le tribunal le respect de la liberté de conscience des enfants et des parents que permettait le choix d'avoir des repas sans porc et « une position de principe se référant à une conception du principe de laïcité ${ }^{18} »$. Il faut se reporter à l'ordonnance du même tribunal du 12 août 2015 - rendue sur la même affaire dans le cadre d'une procédure d'urgence - pour apprendre que cette position de principe apparaissait dans le courrier envoyé aux parents d'élèves par le maire et dans lequel il expliquait fonder sa décision sur des motifs tirés du respect du principe de laïcité. La médiatisation de la décision du maire, à travers notamment le communiqué de presse de la mairie, n'y aura sans doute pas été étrangère. Au final, le tribunal se prononce en sens contraire à la seule affaire précédente comparable ${ }^{19}$ et à la première décision prise dans le cadre de la procédure d'urgence. Dans ce dernier cas, le tribunal avait considéré qu'« eu égard au contenu des menus proposés aux enfants [un seul repas hebdomadaire était concerné] et aux mesures d'information mises en place à l'attention des familles, l'accès aux services de restauration scolaire de l'ensemble des usagers, y compris les enfants de confession musulmane, ne paraît pas compromise [sic]». Cette ordonnance allait finalement dans le sens des préconisations du Défenseur des droits, selon lequel «afin d'éviter tout litige, les mairies qui s'en tiennent au principe de neutralité religieuse en matière de repas scolaires devraient

18. L'on signalera ici qu'en application de l'article R. 625-3 du Code de justice administrative, le tribunal de Dijon avait sollicité la Commission nationale consultative des droits de l'homme qui avait estimé que «la délibération s'appuie sur une interprétation erronée des principes de laïcité et d'égalité et méconnaît l'intérêt supérieur de l'enfant».

19. V. supra, TA Marseille, 26 nov. 1996, Mme Zitouni c/ Commune de Marignane. 
en informer les parents lors de l'inscription à la cantine. Les menus affichés à l'avance doivent pouvoir permettre aux parents de prévoir les jours de présence de leur enfant ${ }^{20} »$.

Il n'est pas certain que le jugement du 28 août 2017 permette de mettre fin aux tentatives ponctuelles d'instrumentalisation des menus de substitution dans les cantines scolaires aux fins d'asseoir une "position de principe se référant à une conception du principe de laïcité ${ }^{21} »$. Il n'en reste pas moins qu'en présence de ce type de prise de position le rappel récurrent, répété, au cadre juridique applicable qui se caractérise essentiellement par l'absence d'obligation des collectivités locales - la très rare jurisprudence allant dans ce sens - peut mener à une impasse. Ce jugement présente au moins l'intérêt d'appréhender ce sujet sous un angle autre que celui de la laïcité, en recherchant l'intérêt supérieur des enfants.

20. Rapport précit., p. 23.

21. V. D. Roman, «Fin des menus de substitution dans les cantines scolaires: "cochon qui s'en dédit"! », AJDA 2017, p. 2207. 